\title{
Experimental Organism Decidualization
}

National Cancer Institute

\section{Source}

National Cancer Institute. Experimental Organism Decidualization. NCI Thesaurus. Code C123637.

A focal lesion within the uterus consisting of markedly hypertrophied stromal cells with cytoplasmic glycogen and prominent nuclei. 